\title{
Anwendertreffen: DeGIR-QS-Register - Was gibt es Neues?
}

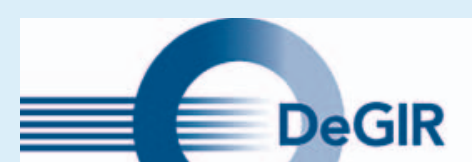

\section{DEUTSCHE GESELLSCHAFT FÜR INTERVENTIONELLE RADIOLOGIE UND MINIMAL-INVASIVE THERAPIE}

\author{
in der Deutschen Röntgengesellschaft e.V.
}

Bei der DeGIR-QS-Software handelt es sich um die größte Qualitätssicherungssoftware einer medizinischen Fachgesellschaft. Aktuell werden pro Jahr ca. 190000 minimalinvasive Eingriffe durch interventionelle Radiologen und Neuroradiologen eingetragen.

Prof Dr. Philipp Paprottka und Prof. Dr. Arno Bücker informieren über Neurungen im
DeGIR-QS-Register, geplante Auswertungen, wie z. B. eine Deutschlandkarte für die flächendeckende Versorgung mit radiologisch durchgeführten gefäßverschließenden Maßnahmen zur interventionellen Behandlung von Blutungen, und neue Projekte, wie z. B. die Nutzbarkeit der Software für prospektive multizentrische Studien.
Anwendertreffen zum DeGIR-QSRegister auf dem 101. RöKo:

Freitag, 22.05.2020, 11:00-12:00 Uhr, Raum Krause 